\title{
ESTIMATIVA DA FORÇANTE RADIATIVA DIRETA DE AEROSSÓIS SOBRE A SUPERFÍCIE EM REGIÃO DE TRANSIÇÃO PANTANAL-CERRADO NO ESTADO DE MATO GROSSO, BRASIL
}

\author{
PALÁCIOS, Rafael da Silva - rafael.pgfa@gmail.com \\ Mestrando - Física Ambiental - Universidade Federal de Mato Grosso / Cuiabá-MT \\ SALLO, Fernando da Silva - fdss88@gmail.com \\ Doutorando - Física Ambiental - Universidade Federal de Mato Grosso / Cuiabá-MT \\ SANTOS, Anna Carolinna Albino - anna.annilorac@gmail.com \\ Mestranda - Física Ambiental - Universidade Federal de Mato Grosso / Cuiabá-MT \\ NOGUEIRA, José de Souza - nogueira@ufmt.br \\ Doutor/Professor - Física Ambiental - Universidade Federal de Mato Grosso / Cuiabá-MT \\ SANTANNA, Francielle Bonfliglio - elektra@fisica.ufmt.br \\ Doutora/Professora - Pós-Graduação em Ciências Ambientais pela Universidade de Cuiabá / Cuiabá-MT.

\begin{abstract}
RESUMO: Estudos das propriedades óticas de aerossóis em escalas locais e suas relações com a radiação solar são de grande importância para a pesquisa atmosférica. No presente trabalho foi estimada a Forçante Radiativa de Aerossóis (ARF) sobre a superfície em uma região de transição pantanal-cerrado no estado de Mato Grosso. A região de estudo é acometida, todos os anos na estação seca, por inúmeros registros de focos de incêndios. Dessa forma buscou-se investigar quantitativamente os valores de ARF para o período seco de 2012 nessa região. Os valores de ARF foram estimados através da diferença entre PAR $_{\text {bac }}$ (Photosynthetically Active Radiation de background ) e a média diária da PAR e os valores de AOD (Aerosol Optical Depth). Os picos negativos de ARF atingiram $-63 \mathrm{Wm}^{-2}$ em meados do mês de setembro onde o número de focos de incêndio ultrapassou 5000 registros, elevando os valores de $A_{0 D} D_{500 n m}$ à 1.4 . A relação entre a $A R F$ e $A O D_{500 n m}$ evidenciou uma queda no fluxo da PAR de $-11.49 \mathrm{Wm}^{-2}$ para a variação de 0.1 nos valores de $A O D_{500 n m}$.
\end{abstract}

PALAVRAS-CHAVE: Profundidade ótica de aerossóis, AERONET, queima de biomassa.

DIRECT RADIOATIVE FORCING ESTIMATION OF AEROSOL OVER THE SURFACE IN PANTANAL TRANSITION REGION - CERRADO IN MATO GROSSO STATE, BRAZIL

ABSTRACT: Understanding aerosol optical properties on local scale and its relation to solar radiation is essential to atmospheric researches. This study aimed to estimate Aerosol Radioactive Forcing (ARF) over a surface on pantanal-cerrado transition region in Mato Grosso state. The region affected by numerous fire outbreaks all year in dry season. Therefore, we sought to investigate, quantitatively, the ARF values for the year 2012 dry season. The ARF values was estimated through the difference between a fixed value of PAR $\mathrm{R}_{\text {bac }}$ (background Photosynthetically Active Radiation) and daily average of PAR and $A O D_{500 \mathrm{~nm}}$ (Aerosol Optical Depth) values. ARF negative peaks reaches $-63 \mathrm{Wm}^{-2}$ in mid-september when the fire outbreaks overtakes 5000 records, increasing $A O D_{500 \mathrm{~nm}}$ values to 1.4. Moreover, high ARF values combined to 0.1 in $A O D_{500 \mathrm{~nm}}$ variation led to a drop in PAR surface flux of $-11.49 \mathrm{Wm}^{-2}$.

KEYWORDS: Aerosol optical depth, AERONET, biomass burning.

\section{INTRODUÇÃO}

Os efeitos provocados pelos aerossóis sobre o meio ambiente são estudados extensivamente em diferentes escalas de espaço e tempo, assim como seus vários efeitos sobre o tempo meteorológico e o clima (MURTHY et al., 2014). As mudanças dos parâmetros atmosféricos e suas relações com a radiação solar impulsionam a pesquisa atmosférica (KUMAR et al., 2013). Aerossóis atmosféricos atuam de forma direta e indireta na transmissão de radiação a superfície terrestre (ZHANG et al., 2013; LI et al., 2014), interferindo no balanço de radiação e consequentemente nos fluxos de superfície e no balanço de energia (BALAKRISHNAIAH et al., 2011; MURTHY et al., 2014). 
A Forçante Radiativa de Aerossóis (ARF), (Radiative Forcing of Aerosols), é um parâmetro essencial na análise dos efeitos diretos dos aerossóis sobre a transmissão de radiação à superfície da terra (RAMANATHAN et al., 2001). Os efeitos de espalhamento e absorção da radiação associam-se, respectivamente, a uma forçante radiativa negativa e positiva sobre a superfície (MENON et al., 2013). Uma das formas de se estimar ARF pode ser feita através da análise da Profundidade de Ótica do Aerossol (AOD), (Aerosol Optical Depth), e sua relação com o fluxo de radiação que incide sobre a superfície (JAYARAMAN et al., 1998).

O cerrado brasileiro contribui com grandes emissões de aerossóis devido à queima de biomassa na estação seca na região Centro-Oeste do Brasil. Nas últimas décadas grande parte da vegetação natural do cerrado tem sido substituída por áreas destinadas às atividades agropecuárias (SANO et al., 2002; SANO et al., 2008), ocorrendo assim uma mudança expressiva nas trocas de energia e massa com a atmosfera (BATLE-BAYER et al., 2010).

No estado de Mato Grosso os efeitos de queimadas naturais devido à sazonalidade agravam ainda mais o efeito dos materiais particulados lançados à atmosfera. O período de seca é relativamente consistente de ano para ano, variando apenas por algumas semanas. 0 período de transição está concentrado entre os meses de agosto e novembro, com algumas variações regionais (SCHAFER et al., 2008).

Os dados de AOD para a região Centro-Oeste do Brasil tanto possibilitam uma caracterização das propriedades óticas da atmosfera local, como auxiliam no entendimento das trocas radiativas através da ARF. Desta forma, o objetivo geral desse trabalho consiste em se estimar quantitativamente os valores da ARF em um período de transição (Seco-Úmida) em uma região de transição pantanal-cerrado no estado de Mato Grosso, estimativa essa feita através da relação entre PAR (Photosynthetically Active Radiation) e AOD, sendo os últimos obtidos junto à rede AERONET (Aerosol Robotic Network), (HOLBEN et al., 1998; HOLBEN et al., 2001).

Os objetivos específicos desse trabalho são: quantificar a ARF sobre a superfície da região de transição pantanal-cerrado através das alterações no fluxo da PAR provocados pelos aerossóis; avaliar os valores estimados para a ARF em função da variabilidade dos valores da AOD para o período de transição entre estação seca e chuvosa na área de estudo.

\section{MATERIAIS E MÉTODOS}

A área de estudo consiste em uma região rural com vegetação típica de cerrado, as características do cerrado no município de Cuiabá (características da baixada cuiabana) apontam para uma formação de um ecossistema com a predominância de floresta com baixa densidade e formação de gramíneas, o chamado campo sujo (FURLEY \& RATTER, 1988; RODRIGUES et al. 2014). Essa classificação para o cerrado da baixada cuiabana é dada exclusivamente por sua estrutura fisionômica cuja característica predominante é herbáceo arbustivo, composto por arbustos esparsos e subarbustos cuja vegetação lenhosa apresenta aproximadamente $2 \mathrm{~m}$ de altura e cobertura menor que $5 \%$.

A Fazenda Miranda, no município de Cuiabá, capital do estado de Mato Grosso, situa-se a aproximadamente $20 \mathrm{~km}$ a sudeste da região urbanizada da capital (latitude 15072' S, longitude 56 $02^{\prime} \mathrm{W}$ e com altitude de $175 \mathrm{~m}$ acima do nível do mar), local onde se encontra o fotômetro solar CIMEL controlado pela rede AERONET e também uma torre micrometeorológica da qual foram utilizados os dados de PAR. A Figura 1 ilustra a área de trabalho detalhando a instrumentação utilizada na pesquisa. As médias anuais de precipitação e temperatura para a área de estudo são de $1200 \mathrm{~mm}$ e $27^{\circ} \mathrm{C}$, respectivamente, com um intervalo de seca bem caracterizado de maio a setembro (BIUDES et al., 2015). Nesse sentido o intervalo de tempo abordado nesse estudo é caracterizado como estação seca, entretanto observa-se que o mês de setembro, último mês da estação seca, nesse trabalho foi considerado um período de transição entre estação seca e chuvosa. 


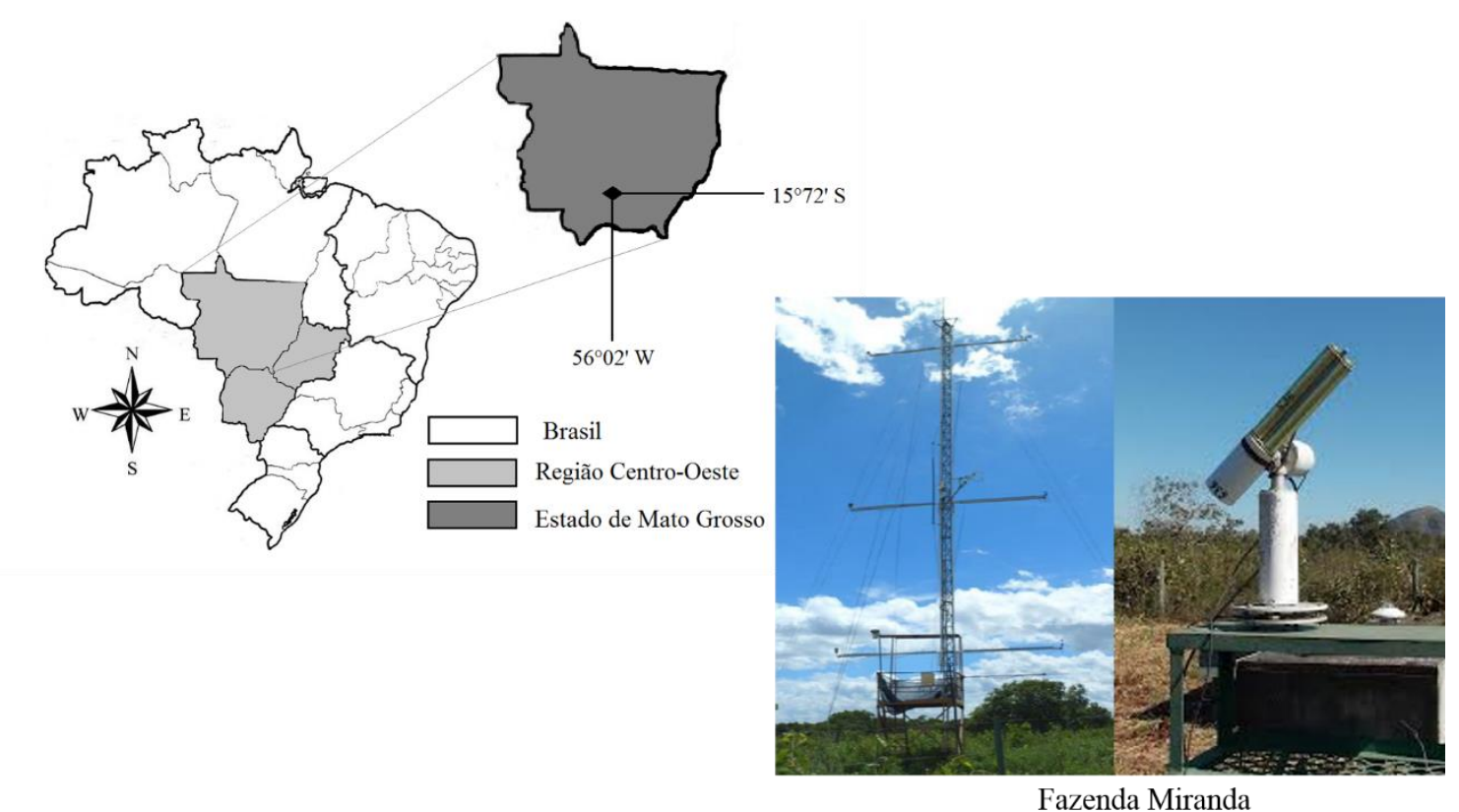

Figura 1. Localização da área de estudo, no detalhe instrumentação na Fazenda Miranda, torre micrometeorológica e o fotômetro CIMEL.

Os dados de AOD, medidos pelo fotômetro solar CIMEL, foram obtidos junta à rede AERONET através do site (http://aeronet.gsfc.nasa.gov/). As informações detalhadas sobre a instrumentação, protocolos de medidas, precisão fotométrica, calibração e métodos de processamento podem ser obtidos em Holben et al. (1998). A rede AERONET disponibiliza os dados de AOD de forma pontual (dados registrados em intervalo de minutos, assincronamente), diária e mensal, em três níveis de qualidade, 1.0, 1.5 e 2.0. O nível 1.0 contém os dados brutos, diretamente das leituras do fotômetro, os dados de nível 1.5, passam por uma triagem, um algoritmo de inversão, onde possíveis dados de dias com nuvens são descartados, já os dados de nível 2.0 além do processamento anterior, recebem um certificado de qualidade fornecido pela própria rede.

No presente trabalho foram utilizados os dados pontuais de profundidade ótica para a radiação na faixa de $500 \mathrm{~nm}$, sendo o arquivo de dados de nível 2.0 , cuja qualidade é certificada pela rede (HOLBEN et al., 1998; SMIRNOV et al., 2000). O período abordado no estudo se concentrou nos meses de julho, agosto e setembro de 2012, uma vez que para esse período há poucos dados descartados pela presença de nuvens, propiciando uma análise quantitativa dos resultados.

As medições de PAR foram realizadas com o sensor LI190SB (LI-COR Biosciences, Inc., Lincon, $\mathrm{NE}, \mathrm{USA}$ ) instalado a $5 \mathrm{~m}$ de altura na torre micrometeorológia, cuja faixa de leitura espectral vai de 400 a $700 \mathrm{~nm}$ com registros a cada 30 minutos. O fotômetro CIMEL e a torre micrometeorológia são ilustrados no detalhe da Figura 1.

Os dados pontuais de $A O D_{500 \mathrm{~nm}}$ foram reorganizados, uma vez que os mesmos foram trabalhos de forma sincronizada com os dados de PAR. O pré-processamento dos dados passou por uma rotina escrita em Fortran 90. Nessa rotina buscou-se a sincronização entre os dados de PAR e $A O D_{500 n m}$, uma vez que as medidas de PAR são registradas a cada 30 min e os registros de $A O D_{500 \mathrm{~nm}}$ são pontuais. Para cada valor de PAR foi realizada uma regressão com os valores pontuais de $A O D_{500 n m}$ para esse intervalo de tempo através de interpolação linear.

Após a sincronização dos dados foi realizada a média diária tanto da $A_{0} D_{500 n m}$ como da PAR. A média foi realizada das $08: 00$ as 16:00 horas (horário local) afim de se avaliar as influências diárias dos valores de $A O D_{500 \mathrm{~nm}}$ nas variações de PAR. Para os valores de $A O D_{500 \mathrm{~nm}}$ abaixo de 0.2 , ou seja, valores de $A O D_{500 \mathrm{~nm}}$ até o dia do ano 220 , foi feita uma média sendo esse valor considerado valor de background para $A_{0 D} D_{500 \mathrm{~nm}}$. A escolha para esse valor de 
background foi feita observando-se a baixa variação dos valores de $A O D_{500 n m}$ para os primeiros dias do mês de julho.

A estimativa da ARF diária foi obtida através da diferença entre o valor médio da PAR para os valores de AOD abaixo do valor de background $\left(P A R_{b a c}\right)$ e os valores médios diários da PAR, desta forma e queda dos valores de fluxo de radiação seriam provocados apenas por variações nos valores de $A_{0 D}$ 500nm (PROCÓPIO et al., 2004).

$$
A R F=P A R_{b a c}-P A R
$$

Os valores obtidos de ARF foram analisados em função dos valores de $A_{0} D_{500 n m}$, obtendo assim, um modelo para os valores diários de ARF em função de $A O D_{500 n m}$, o modelo obtido foi comparado ao proposto por Procópio et al. (2004).

No tratamento estatístico dos dados foram testadas às normalidades das distribuições, os valores de ARF estimados foram comparados com os valores modelados em função da $A O D_{500 n m}$ e com os valores obtidos pelo modelo proposto por Procópio et al. (2004). A correlação entre os dados foi representada pelo coeficiente de correlação de Spearman (PALÁCIOS et al., 2014). A determinação de tais coeficientes foi realizada mediante a técnica de Bootstrap com um total de 10000 reamostragens.

\section{RESULTADOS E DISCUSSÃO}

Os valores diários de $A O D_{500 n m}$ apresentam uma alta taxa de variação no período analisado, a Figura 2 representa a variação nos valores de $A O D_{500 n m}$ em função do dia do ano, percebe-se que até o dia de número 220 os valores permanecem abaixo de 0.2 , a partir desse, aumentam gradativamente até o dia 255 onde atingem 1.4, voltando a diminuir a partir desse dia. Com relação ao aumento gradativo nos valores de $A_{0 D} D_{500 n m}$, Procópio et al. (2004) ressalta que para toda região central do Brasil as características da AOD estão relacionadas diretamente com os registros de focos de queimadas, que atingem picos máximos nos meses de agosto e setembro devido ao regime de seca na região.

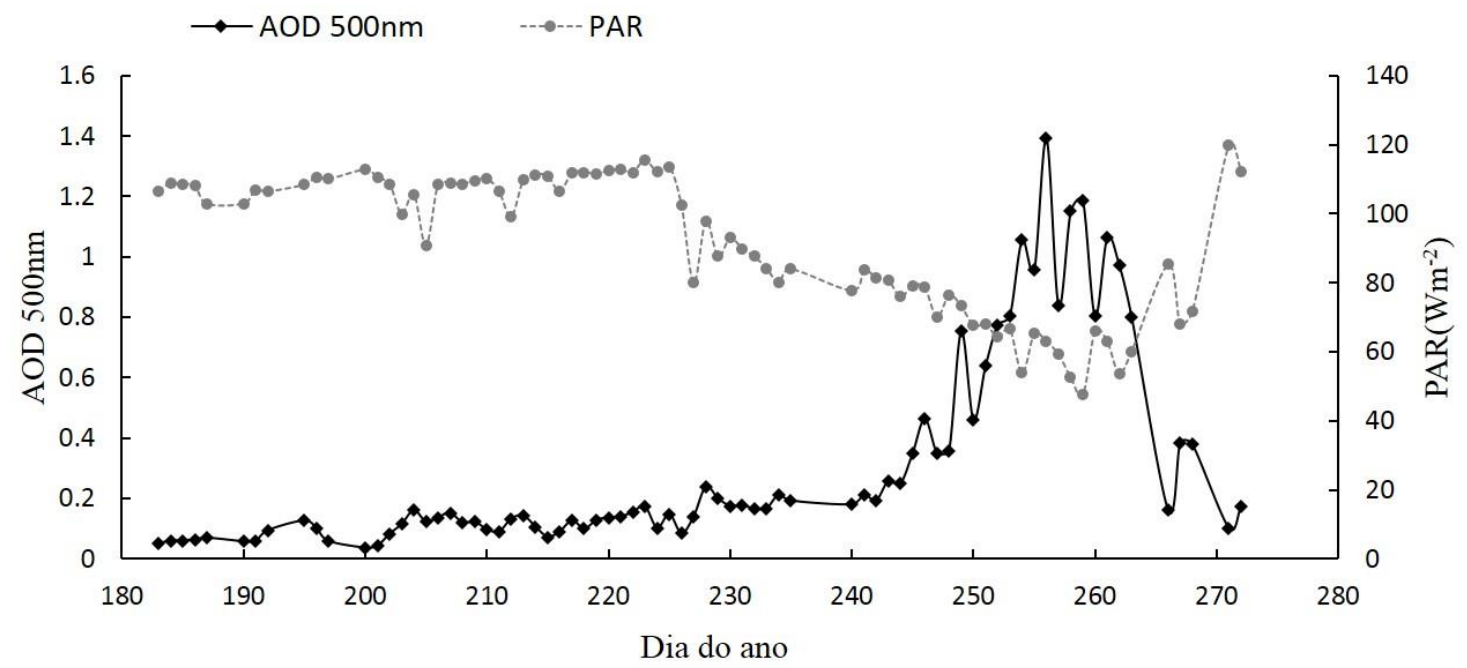

Figura 2. Distribuição dos valores de $P A R$ e $A O D_{500 n m}$ com relação aos dias do ano.

Ainda com relação a Figura 2, o comportamento da PAR em função do dia do ano é praticamente inverso ao da AOD, os valores de PAR diminuem a medida que os valores de AOD aumentam, mostrando a evidente influência da AOD nas variações do fluxo de radiação sobre a superfície. A queda no fluxo de radiação está diretamente ligada a queima de biomassa que gera grandes quantidades de carbono negro (black carbon), (ANDREAE et al., 2004; ARTAXO et al., 2006) forte absorvedor de radiação, no entanto, a de se levar em 
consideração o efeito de dispersão da luz, que é predominante nas partículas de fumaça conforme calculado por Procópio et al. (2003).

Os valores de ARF (estimados através da equação 1 ) em função dos valores de $A O D_{500 n m}$ estão representados na Figura 3, através da equação de segundo grau proposta pela relação, pode-se obter os valores de ARF simplesmente levando-se em consideração os valores de AOD $_{500 n m}$.

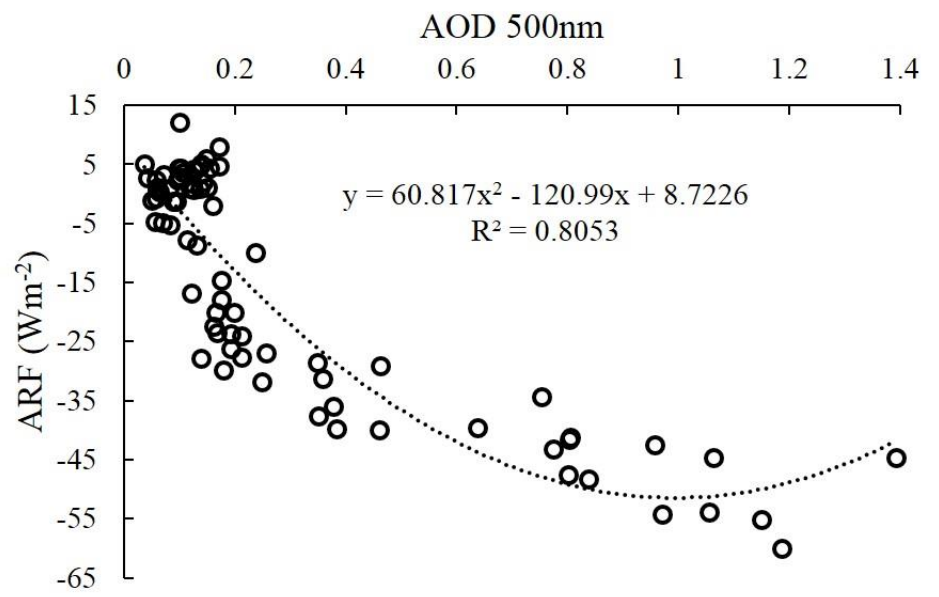

Figura 3. Distribuição dos valores estimados de ARF em função da AOD 500 nm, curva ajustada para a distribuição de pontos.

O trabalho de Procópio et al. (2004) ajusta uma equação também de segundo grau, para relacionar os valores de ARF sobre a superfície em uma região de transição Amazôniacerrado, essa equação fornece os valores de ARF_model2 mostrados na Figura 4. A Figura 4 representa as distribuições de ARF estimadas através da equação 1 , os valores de ARF_model1, estimados através da equação ajustada pela distribuição da Figura 3 e os valores de $\overline{A R F}$ _model2, estimados através do modelo proposto por Procópio et al. (2004).

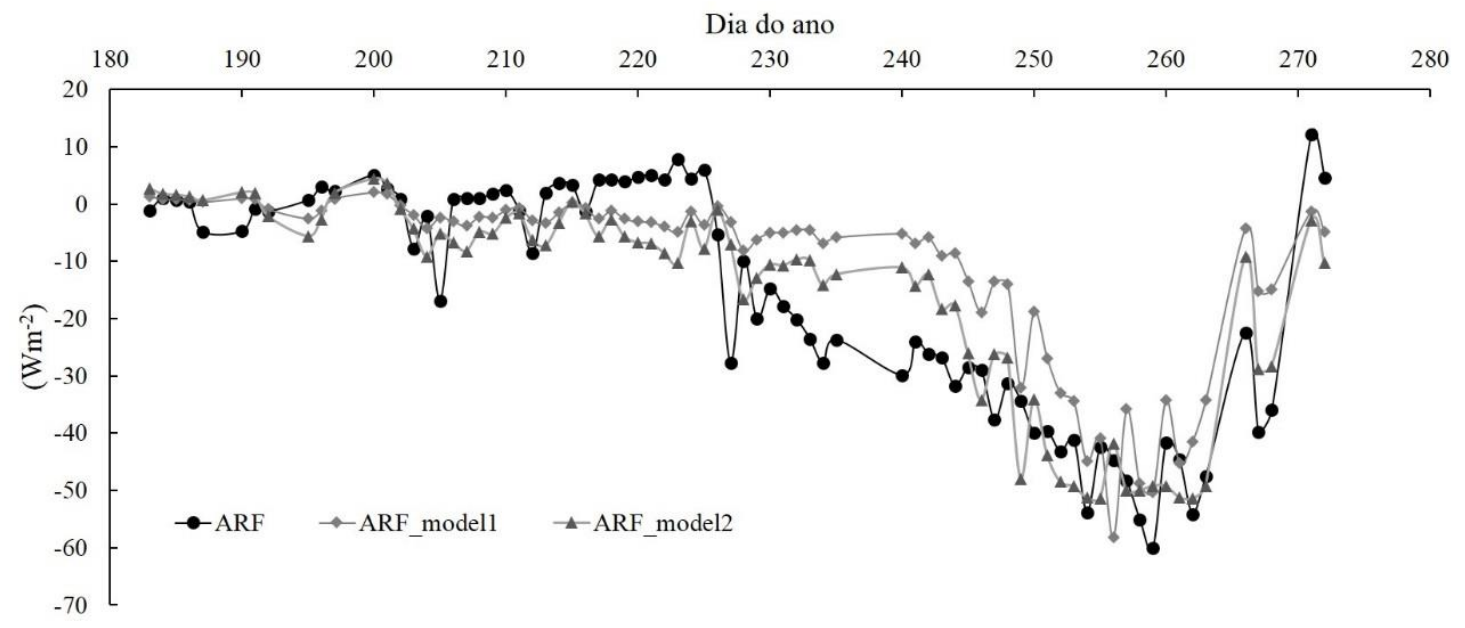

Figura 4. Distribuição dos Valores de ARF, ARF_model1 e ARF_model2 em função do dia do ano para o período de estudo.

A Tabela 1 representa as correlações realizadas para os valores apresentados na Figura 4. Os coeficientes obtidos mostram uma correlação estatisticamente significativa para todos os conjuntos de dados observados. A correlação entre os valores de ARF_model1 e ARF_model2 já era esperada uma vez que ambos modelos foram ajustados por curvas de equações de segundo grau. 
Tabela 1. Coeficientes de Spearman, erros, desvios e intervalos de confiança para as medidas de ARF, ARF_model1 e ARF_model2.

\begin{tabular}{|c|c|c|c|c|c|}
\hline \multicolumn{3}{|c|}{ Correlação de Spearman } & ARF & ARF_model1 & ARF_model2 \\
\hline \multirow[t]{6}{*}{ ARF } & \multicolumn{2}{|c|}{ Coeficiente de correlação } & 1.000 & 0.785 & 0.783 \\
\hline & \multicolumn{2}{|c|}{$\mathrm{N}$} & 76 & 76 & 76 \\
\hline & Bootstrap & Desvio & 0.000 & -0.007 & -0.007 \\
\hline & & Erro & 0.000 & 0.067 & 0.066 \\
\hline & $\begin{array}{l}\text { Intervalo de } \\
\text { confiança }\end{array}$ & Inferior & 1.000 & 0.629 & 0.628 \\
\hline & $95 \%$ & Superior & 1.000 & 0.886 & 0.882 \\
\hline \multirow[t]{6}{*}{ ARF_model1 } & \multicolumn{2}{|c|}{ Coeficiente de correlação } & 0.785 & 1.00 & 0.996 \\
\hline & \multicolumn{2}{|c|}{$\mathrm{N}$} & 76 & 76 & 76 \\
\hline & Bootstrap & Desvio & -0.007 & 0.000 & -0.001 \\
\hline & & Erro & 0.067 & 0.000 & 0.004 \\
\hline & $\begin{array}{l}\text { Intervalo de } \\
\text { confiança }\end{array}$ & Inferior & 0.629 & 1.000 & 0.984 \\
\hline & $95 \%$ & Superior & 0.886 & 1.000 & 1.000 \\
\hline \multirow[t]{6}{*}{ ARF_model2 } & \multicolumn{2}{|c|}{ Coeficiente de correlação } & 0.783 & 0.996 & 1.00 \\
\hline & \multicolumn{2}{|c|}{$\mathrm{N}$} & 76 & 76 & 76 \\
\hline & Bootstrap & Desvio & -0.007 & -0.001 & 0.00 \\
\hline & \multirow{3}{*}{$\begin{array}{l}\text { Intervalo de } \\
\text { confiança } \\
95 \%\end{array}$} & Erro & 0.066 & 0.004 & 0.000 \\
\hline & & Inferior & 0.628 & 0.984 & 1.000 \\
\hline & & Superior & 0.882 & 1.000 & 1.000 \\
\hline
\end{tabular}

Bootstrap com 10000 reamostragens, nível de significância de 0.01 .

Com relação aos de ARF_model1 e os valores de ARF, a correlação entre ambos mostra a eficiência do modelo proposto na análise do forçamento radiativo escrito somente em função da $A_{0} D_{500 \mathrm{~nm}}$. Como se observa na Tabela 1 o coeficiente de Spearman para ARF e ARF_model1 foi de 0.785 em um intervalo de 0.629 a 0.886 , apresentando um erro de 0.067 .

Os dados apresentados na Figura 4 e descritos estatisticamente na Tabela 1, retratam as variações de valores de $A R F$, influenciados diretamente por variações de $A O D_{500 n m}$. $A$ literatura atual retrata vários trabalhos sobre as variações de AOD, assim como de outros parâmetros físicos associados as propriedades óticas de aerossóis (KUMAR et al., 2013; ZHANG et al., 2013; LI et al., 2014), no entanto há uma carência de trabalhos relacionados ao forçamento radiativo de aerossóis, principalmente sobre a superfície (IPCC, 2007).

As quedas nos valores de PAR alteram todo o balanço de energia sobre a superfície, uma vez que os mesmos alteram diretamente os fluxos de superfície como calor sensível e calor latente (MURTHY et al., 2014). Com relação aos valores obtidos para ARF, Procópio (2005) estimou variações de -26 a $-62 \mathrm{Wm}^{-2}$ sobre a superfície em uma região de transição Amazônia-cerrado, enquanto que os valores estimados nesse trabalho variam de 10 a $-63 \mathrm{Wm}^{-}$ 2 para a região de transição pantanal-cerrado.

Os valores positivos de ARF se devem ao método empregado, nesses casos os valores de PAR média diária foram menores que os valores de $P A R_{b a c}$. $O$ trabalho de Li et al. (2014) analisando a relação entre AOD e o crescimento higroscópico dos aerossóis sobre a Ásia Oriental em 2006 encontrou quedas nos fluxos de radiação que variaram de -9 a $-29 \mathrm{Wm}^{-2}$ devido principalmente a aerossóis de sulfatos.

Para a região de estudo, a sazonalidades dos padrões meteorológicos influenciam assim como no trabalho de Kumar et al. (2013), a qualidade e a distribuição de tamanho de aerossóis, o agravante para a região central do Brasil são os elevados registros de focos de incêndio que ocorrem principalmente nos meses de agosto e setembro, conforme Palácios et al. (2014). Diferentemente do trabalho de Kumar et al. (2013) onde os maiores registros de 
$\mathrm{AOD}_{500 \mathrm{~nm}}$ ocorrem na primavera e não ultrapassam 0.4 , na área de estudo os maiores valores registrados atingem 1.4 , justamente no período de seca onde os registros de focos de incêndio ultrapassaram 5000 no mês de setembro de 2012 .

As características do tipo de aerossol presente na área de estudo ainda devem ser analisadas assim como as contribuições dos efeitos desses aerossóis de forma indireta, atuando como núcleos de condensação (PRENNI et al., 2009), no entanto a sazonalidade característica da região aponta para uma formação típica de queima de biomassa.

A relação entre $A R F$ e $A O D_{500 n m}$ mostra que a variação de 0.1 na $A O D_{500 \mathrm{~nm}}$ implica em um incremento de $-11.49 \mathrm{Wm}^{-2}$ no valor de $A R F$, desta forma para valores críticos de $A O D_{500 \mathrm{~nm}}$ atingidos no período de seca, nessa região, explicam de forma satisfatória a queda nos valores de PAR.

A variabilidade dos valores de $A O D_{500 n m}$ é claramente explicada pelo aumento significativo dos registros de focos de queimadas no estado de Mato Grosso no período analisado (Figura 5), conforme Palácios et al. (2014) a grande exposição da área de estudo à seca proporciona elevados registros de focos de queimadas em toda região de cerrado no Brasil nos meses de agosto e setembro. A Figura 5 evidencia a correlação entre os focos de queimadas para o estado de Mato Grosso com os valores de $A O D_{500 \mathrm{~nm}}$ para os meses analisados. Para esse período não houve registros de precipitação o que explica tanto a elevação dos registros de focos de queimadas como o aumento da profundidade ótica dos aerossóis e o forçamento radiativo sobre o fluxo da PAR.

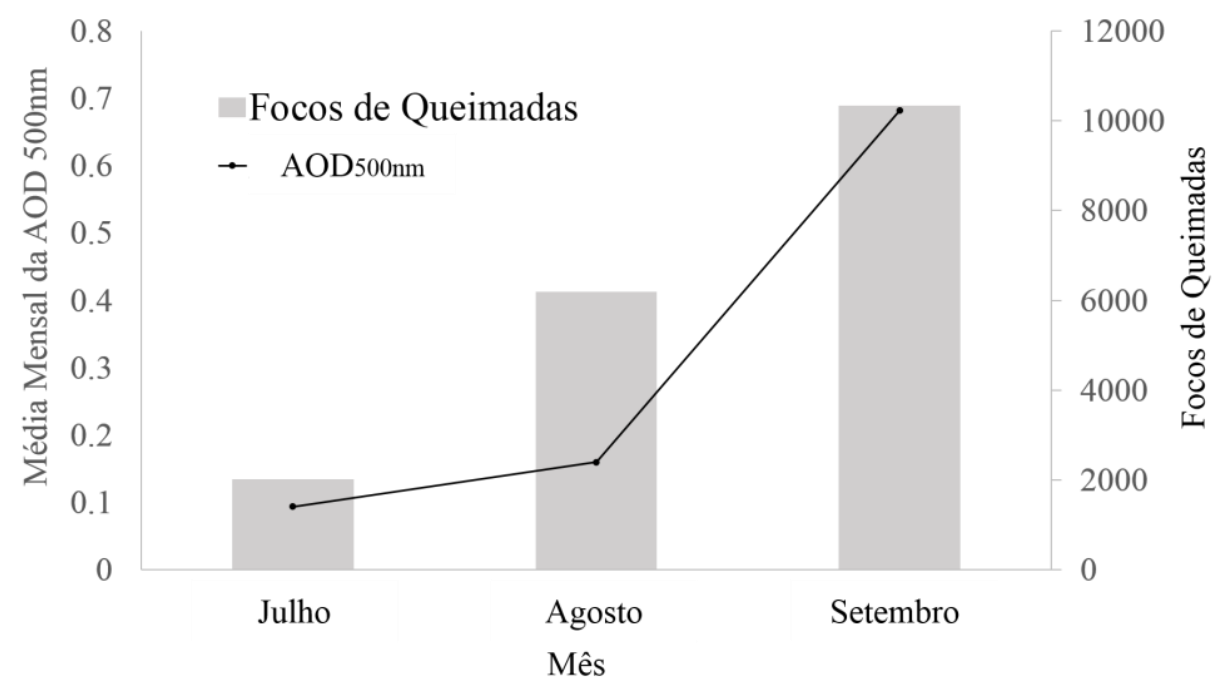

Figura 5. Relação entre os registros mensais de focos de queimadas para o estado de Mato Grosso com as médias mensais da $A_{0 D} D_{500 \mathrm{~nm}}$ para os meses de julho, agosto e setembro de 2012.

\section{CONCLUSÃO}

O presente trabalho apresenta a estimativa da ARF sobre a superfície em uma região de transição pantanal-cerrado no estado de Mato Grosso. Os valores de ARF estimados para a região de estudo atingiram $-63 \mathrm{Wm}^{-2}$ para meados do mês de setembro, justamente o mês que teve elevados registros de focos de incêndio (mais de 5000 registros). Através dos valores estimados de ARF e os valores de AOD $500 \mathrm{~nm}$ foi ajustada uma equação de segundo grau capaz de descrever os valores do forçamento radiativo simplesmente através da AOD. O modelo ajustado para os dados de ARF ainda foi correlacionado ao modelo, também de superfície, proposto por Procópio et al. (2004), a correlação entre tais modelos apresentou um coeficiente de correlação de Spearman de 0.996. Para a relação entre a ARF e $A O D_{500 n m}$ a variação de 0.1 nos valores de $A O D_{500 n m}$ provoca uma diminuição de $-11.49 \mathrm{Wm}^{-2}$ no fluxo da PAR. 


\section{AGRADECIMENTOS}

Agradecemos a CAPES, pelo apoio financeiro, ao grupo de pesquisa do Programa de Pósgraduação em Física Ambiental e ao grupo de estudos do Instituto de Física da USP pela utilização dos dados da rede AERONET.

\section{REFERÊNCIAS BIBLIOGRÁFICAS}

ANDREAE, M.O., ROSENFELD, D., ARTAXO, P., COSTA, A.A., FRANK, G.P., LONGO, K.M., SILVA-DIAS, M.A.F. Smoking Rain Clouds over the Amazon. Science, v. 303, p. 1337-1342, 2004.

ARTAXO, P., OLIVEIRA, P.H., LARA, L.L., PAULIQUEVIS, T.M., RIZZO, L.V., JUNIOR, C.P., PAIXÃO, M.A., LONGO, K.M., FREITAS, S., CORREIA, A. Efeitos climáticos de partículas de aerossóis biogênicos e emitidos em queimadas na Amazônia. Revista Brasileira de Meteorologia, v. 21, p. 168-189, 2006.

BALAKRISHNAIAH, G., KUMAR, K.R., KUMAR REDDY, B.S.K., GOPAL, K.R., REDDY, R.R., REDDY, L.S.S., AHAMMED, Y.N., NARASIMHULU, K., MOORTHY, K.K., BABU, S.S. Analysis of optical properties of atmospheric aerosols inferred from spectral AODs and Ångström wavelength exponent. Atmospheric Environment, v. 45, p. 1275-1285, 2011.

BATLLE-BAYER, L.; BATJES, N.H; BINDRABAN, P.S. Changes in organic carbon stocks upon land use conversion in the Brazilian Cerrado: A review. Agriculture, Ecosystems and Environment, v. 137, p. 47-58, 2010.

BIUDES, M.S., VOURLITIS, G.L., MACHADO, N.G., ARRUDA, P.H.Z., NEVES, G.A.R., LOBO, F.A., NEALE, C.M.U., NOGUEIRA, J.S. Patterns of energy exchange for tropical ecosystems across a climate gradient in Mato Grosso, Brazil. Agricultural and Forest Meteorology, v. 202, p. 112-124, 2015.

FURLEY, P. A.; J. A. RATTER. Soil resources and plant communities of the central Brazilian cerrado and their development, Journal Biogeography, v. 15, p. 97-118, 1988.

HOLBEN, B.N., ECK, T.F., SLUTSKER, I., TANRÉ, D., BUIS, J.P., SETZER, A., VERMOTE, E., REAGAN, J.A., KAUFMAN, Y.J., NAKAJIMA, T., LAVENU, F., JANKOWIAK, I., SMIRNOV A. AERONET - a federated instrument network and data archive for aerosol characterization. Remote Sensing of the Environment, v. 66, n. 1, p. 1-16, 1998.

HOLBEN, B.N., TANRE, D., SMIRNOV, A., ECK, T.F., SLUTSKER, I., ABUHASSAN, N., NEWCOMB, W.W., SCHAFER, J.S., CHANTENET, B., LAVENU, F., KAUFMAN, Y.J., VANDE CASTLE, J., SETZER, A., MARKHAM, B., CLARK, D., FROUIN, R., HALTHORE, R., KARNELI, A., O'NEILL, N.T., PIETRAS, C., PINKER, R.T., VOSS, K., ZIBORDI, G. An emerging ground-based aerosol climatology: aerosol optical depth from AERONET. Journal of Geophysical Research, v. 106, n. D11, p. 12067-12097, 2001.

INPE - Instituto Nacional de Pesquisas Espaciais, 2012. Portal do Monitoramento de Queimadas e Incêndios. Disponível em http://www.inpe.br/queimadas. Acesso em: $17 / 02 / 2014$.

IPCC - Intergovernmental Panel on Climate Change (IPCC). Climate change. The physical science basis: Contribution of Working Group I to the Fourth Assessment Report of the Intergovernmental Panel on Climate Change, p. 129, Chapter 2, 2007.

JAYARAMAN, A., LUBIN, D., S. RAMACHANDRAN, RAMANATHAN, V., WOODBRIDGE, E., COLLINS, W.D., ZALPURI, K.S. Direct observations of aerosol radiative forcing over the tropical Indian Ocean during the January-February 1996 pre-INDOEX cruise. Journal of Geophysical Research, v. 103, p. 13827-13836, 1998. 
KUMAR, K.R., SIVAKUMAR, V., REDDY, R.R., GOPAL, K.R., ADESINA, A.J. Inferring wavelength dependence of AOD and Ångström exponent over a sub-tropical station in South Africa using AERONET data: Influence of meteorology, long-range transport and curvature effect. Science of the Total Environment, v. 461-462, p. 397-408, 2013.

LI, J., HAN, Z., ZHANG, R. Influence of aerosol hygroscopic growth parameterization on aerosol optical depth and direct radiative forcing over East Asia. Atmospheric Research, v. 140-141, p. 14-27, 2014.

MENON, H.B, SHIRODKAR, S., KEDIA, S., RAMACHANDRAN, S., BABU, S., MOORTHY, K.K. Temporal variation of aerosol optical depth and associated shortwave radiative forcing over a coastal site along the west coast of India. Science of the Total Environment, v. 468-469, p. 83-92, 2013.

MURTHY, B.S., LATHA, R., MANOJ KUMAR, MAHANTI, N.C. Effect of aerosols on evapotranspiration. Atmospheric Environment, v. 89, p. 109-118, 2014.

PALÁCIOS, R.S., SALLO, F.S., do PRADO, M.J., de MUSIS, C.R., NOGUEIRA, J.S. Análise da profundidade ótica de aerossóis e coeficiente de Angstron no Cerrado Mato-grossense. Revista Brasileira de Climatologia, v. 14, p. 173-185, 2014.

PRENNI, A.J., PETTERS, M.D., KREIDENWEIS, S.M., HEALD, C.L., MARTIN, S.T., ARTAXO, P., GARLAND, R.M., WOLLNY, A.G., PÖSCCHL, U. Relative roles of biogenic emissions and Saharan dust as ice nuclei in the Amazon basin. Nature Geoscience, v. 2, p. 402-405, 2009.

PROCOPIO, A. S., REMER, L.A., ARTAXO, P., KAUFMAN, Y.J., HOLBEN, B.N. Modeled spectral optical properties for smoke aerosols in Amazonia, Geophysical Research Letters, v.30, NO. 24, 2265, 2003.

PROCÓPIO, A.S. Forçante radiativa direta dos aerossóis na Região Amazônica devido à queima de biomassa. 2005. 224 f. Tese (Doutorado) - Instituto de Astronomia, Geofísica e Ciências Atmosféricas da Universidade de São Paulo, São Paulo, 2005.

PROCOPIO, A.S., ARTAXO, P., KAUFMAN, Y.J., REMER, L.A., SCHAFER, J.S., HOLBEN, B.N. Multiyear analysis of amazonian biomass burning smoke radiative forcing of climate. Geophysical Research Letters, v. 31, L03108, p. 1-4, 2004.

RAMANATHAN, V., CRUTZEN, P. J., KIEHL, J. T., ROSENFELD, D., Aerosols, Climate, and the Hydrological Cycle. Science, v. 294 n. 5549 p. 2119-2124, 2001.

RODRIGUES, T. R.; G. L. VOURLITIS; F. D. A. LOBO; R. G. DE OLIVEIRA; J. D. S. NOGUEIRA. Seasonal variation in energy balance and canopy conductance for a tropical savanna ecosystem of south central Mato Grosso, Brazil. Journal Geophysical Research Biogeosciences, v. 119, p. 1-13, 2014.

SANO, E.E.; BARCELLOS, A.O.; BEZERRA, H.S. Assessing the spatial distribution of cultivated pastures in the Brazilian savanna. Pasturas Tropicales, v. 22, p. 2-15, 2002.

SANO, E.E.; ROSA, R.; BRITO, J.L.S.; FERREIRA, L.G. Mapeamento semidetalhado do uso da terra do Bioma Cerrado. Pesquisa Agropecuária Brasileira, v. 43, p. 153-156, 2008.

SCHAFER, J.S.; ECK, T.F.; HOLBEN, B.N.; ARTAXO, P.; DUARTE, A. F. Characterization of the optical properties of atmospheric aerosols in Amazônia from long-term AERONET monitoring (1993-1995 and 1999-2006). Journal of Geophysical Research, v.113, D04204, p. 1-16, 2008. 
SMIRNOV, A., HOLBEN, B.N., ECK, T.F., DUBOVIK, O., SLUTSKER, I. Cloud screening and quality con- trol algorithms for the AERONET data base. Remote Sensing of the Environment, v.73, p. 337-349, 2000.

ZHANG, Z., WENIG, M., ZHOU, W., DIEHL, T., CHAN, K., WANG, L. The contribution of different aerosol sources to the Aerosol Optical Depth in Hong Kong. Atmospheric Environment, v. 83, p. $145-154,2014$.

Texto submetido à RBClima na data de 10/02/2015 